\title{
Encuentros y desencuentros filosóficos y políticos entre Badiou y Rancière
}

\section{Philosophical and Political Agreements and Disagreements between Badiou and Rancière}

\author{
LEONARDO COLELLA \\ Consejo Nacional de Investigaciones \\ Cientificas y Técnicas (Argentina)
}

Recibido: 15/III/2014 Aceptado definitivamente: 10/IV/2014

\section{RESUMEN}

El objetivo de este artículo es contrastar las posturas filosóficas y políticas de Alain Badiou y Jacques Rancière, y aplicar los resultados de ese análisis al ámbito educativo. Para ello abordamos inicialmente las diferencias entre ambos autores respecto de sus teorías del sujeto, sus relaciones con Platón y sus propuestas ontológicas. A continuación consideramos, en primer lugar, una coincidencia acerca del carácter prescriptivo del concepto de «igualdad», y en segundo lugar, una divergencia respecto del «sujeto» y de las consecuencias disruptivas acontecidas a partir de la declaración igualitaria en la educación.

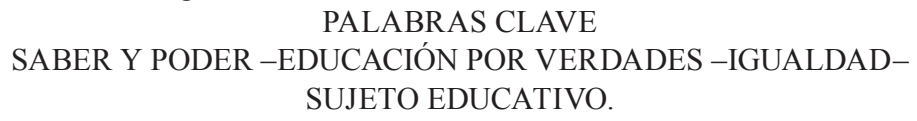

PALABRAS CLAVE

SABER Y PODER -EDUCACIÓN POR VERDADES -IGUALDADSUJETO EDUCATIVO.

\begin{abstract}
The aim of this paper is to contrast the philosophical and political positions of Alain Badiou and Jacques Rancière, and apply the results of this review to education. For this, we initially regard the differences between the two authors about their theories of the subject, its relations with Plato and their ontological proposals. Next, we considered, firstly, a coincidence about the prescriptive concept of «equality», and secondly, a difference with respect to the «subject» and the disruptive consequences from the equalitarian statement in education.
\end{abstract}

\section{KEYWORDS}

\section{KNOWLEDGE AND POWER -EDUCATION BY TRUTHS -EQUALITY- SUBJECT OF EDUCATION}

(C) Contrastes. Revista Internacional de Filosofía, vol. XX № 2 (2015), pp. 217-230. ISSN: 1136-4076 
El objetivo de este ARTículo es contrastar las posturas de Alain Badiou y Jacques Rancière respecto de diversas cuestiones filosóficas, políticas y educativas. En un primer momento, abordaremos ciertas diferencias de orden político que encuentran sustento en sus diversas perspectivas filosóficas sobre ontología y teoría del sujeto. En segundo lugar, nos centraremos en los enfoques educativos que ambos autores han inspirado, la «educación por verdades» de Alain Badiou y la «emancipación intelectual» de Jacques Rancière. En tal sentido, postularemos que en ambos enfoques existe un elemento común: la noción de «igualdad». Finalmente, analizaremos las coincidencias y las divergencias tratadas previamente, situadas en el contexto que dio origen a sus obras, y las vincularemos a escenarios políticos y educativos.

\section{BADIOU Y RANCIÈRE: COINCIDENCIAS Y DIVERGENCIAS FILOSÓFICAS Y POLÍTICAS}

Si indagamos en las obras de Alain Badiou y Jacques Rancière, podremos encontrar algunas divergencias entre ambos autores, incluso exploradas por ellos mismos en algunos de sus textos. En primer lugar, Badiou imputa a Rancière el hecho de evitar el término «Estado», sustituyéndolo por «policía» o algunas otras variantes (Badiou 1998, 92). La sospecha radica en que Rancière eludiría cualquier crítica directa hacia el Estado parlamentario para protegerse de la acusación de «antidemócrata». Es cierto que cuando Badiou realiza estas críticas en la década de 1990, gran parte de la obra política de Rancière aún no estaba desarrollada. Pero aun teniendo en cuenta el posible anacronismo, Rancière despliega un extenso argumento en Le Mésentente. Politique et philosophie (1995), acerca de los motivos por los que elige el término «policía» y no el de Estado. En este sentido, asume ciertos aspectos de la noción de «poder» foucaultiano, y considera que la «policía» sobrepasa, aunque también incluye, los aparatos de Estado. De este modo, Rancière no eludiría la palabra Estado, sino que la subsumiría a su concepto de policía.

Badiou también resalta el hecho de que en Le Mésentente, la propuesta de una política no sea plasmada en forma afirmativa. Señala que Rancière destaca aquello que «no» debe fundamentar la política: la demagogia consensual o la experiencia radical del Holocausto. De esta forma, no se ocuparía centralmente de una definición propositiva de política sino, más bien, de la descripción de los aspectos de su ausencia.

En términos políticos, la principal diferencia radicaría en que para Rancière pareciera ser que la política no es del orden del proyecto organizado o de la prescripción, sino del tono de una declaración de la igualdad en tanto circunstancia histórica, hecho que lo distanciaría del miembro fundador de la Organización Política. Vemos así cómo la política, que se juega entre la potencia de las partes contadas y no contadas, que para Badiou es una cuestión 
estrictamente ontológica, para Rancière sería parte de una fenomenología historicista de una circunstancia igualitaria.

Tal es así que esta diferencia política se traduce, además, en una diferencia filosófica. Badiou señala que Rancière no precisa de una ontología porque inscribe su producción en un estilo antifilosófico. En este aspecto, su obra, para Badiou, consistiría en una ligera variación del antiplatonismo del siglo XX.

Rancière, por su parte, alude a esta crítica en «El uso de las distinciones» (2004). Señala que mientras Badiou asocia el concepto de democracia al de consenso, y opone a ello una política como potencia heterogénea del suplemento acontecimiental, él identifica esa potencia de lo heterogéneo con el démos, y en consecuencia, opone «democracia» a «consenso». En efecto, para Rancière, no hay un principio ontológico de la diferencia política. No existirían, tampoco, sujetos políticos constituidos al amparo de un suplemento ontológico asociado al orden del acontecimiento y de la verdad sino, más bien, sujetos políticos definidos a partir de los modos de subjetivación que transforman los espacios comunes.

No existe, reconoce el propio Rancière, un sujeto cuya tarea sea portar aquella potencia ontológica disruptiva de la excepción que constituyen las verdades:

En política, es el démos como abolición de toda arkhè, de toda correspondencia entre los lugares de gobernante y de gobernado y una «disposición» a ocupar estos lugares. El uno-de-más es la potencia de lo indistinto que deshace las particiones recusando la fijeza de los lugares de lo mismo y de lo otro. No hay entonces sujeto que tenga como propia la potencia de ruptura o de desconexión, no hay sujeto que ejerza una potencia ontológica de la excepción. La excepción es siempre ordinaria (Rancière 2004, 6).

Rancière entonces retorna las críticas por el mismo canal. Destaca en Badiou (pero también en Zizek, Agamben, Negri, Lyotard, Arendt y Milner, cada uno con sus particularidades) la necesidad de recurrir a una potencia ontológica, justificadora del disenso, que desborde la conducta regular de la experiencia consensual. Esta diferencia respecto de diversos autores contemporáneos, Rancière la atribuye a una cuestión pragmática y metodológica: él se ocupó de revisar los archivos obreros, y su filosofía precisaba la invención de conceptos que dieran cuenta de lo que en ellos sucedía.

Sucede que, mientras se operaban estos encuentros con la superpotencia y estos desvíos de la superpotencia, yo estaba ocupado en otra parte, en otra cosa. Trataba entonces de comprender la potencia de algunas palabras como proletario o emancipación. Trabajaba sobre los encuentros, fronteras y pasajes que tuvieron el efecto de separar individuos de la esfera de la experiencia sensible que les estaba asignada (Ibid., 7).

El rechazo a la ontología del suplemento, para Rancière, fue una de- 
cisión consecuente de inscribirse en otra idea de filosofía. Ésta se ocupa más bien de la afirmación (y de la exigencia de verificación) de la igualdad de las inteligencias, de las «reparticiones» de los lugares asignados, de la democracia y la disolución del arkhé, del régimen estético del arte, etc. La filosofía, en términos de Rancière, es indisoluble de una práctica igualitaria, anarquista y creadora, en la medida que es asumida como una actividad crítica con respecto a la policía de los dominios conceptuales y a las particiones instituidas que permitirían trazar sus límites y tornarlos pensables y modificables.

Me he visto conducido entonces a considerar que mi rechazo a ontologizar un principio de lo heterogéneo, mi rechazo de las ontologías de la superpotencia no era una capitulación vergonzosa ante los deberes de la filosofía o el ejercicio parasitario de la histérica viviendo de la deconstrucción del discurso del amo, sino el ejercicio consecuente de otra idea de la filosofía (Ibid., 8).

Una vez identificadas las diferencias reconocidas por ambos autores, sobre las cuestiones ontológicas, sobre el platonismo y el antiplatonismo, el estilo filosófico o antifilosófico y la organización de las consecuencias disruptivas, abordaremos a continuación lo que consideramos un acuerdo sustancial entre ambos filósofos, referido al principio igualitario de las situaciones educativas. Como analizaremos a continuación, estas semejanzas provienen de un contexto particular común que incluyen las experiencias políticas de China a partir de la década de 1960 y los propios sucesos franceses de 1968. Sin embargo, si bien las motivaciones son análogas, algunas distinciones del orden filosófico y político, mencionadas anteriormente, derivan en diferencias en sus posturas educativas, que aunque no podrían considerarse en extremo sustanciales, convienen ser señaladas.

\section{EDUCACIÓN POR VERDADES Y EDUCACIÓN POR LA IGUALDAD}

Más allá de la lógica tradicional, la educación abordada desde una perspectiva badiouiana, traza un vínculo particular con las «verdades». Esto significa que una de sus tareas principales es la de distinguir esas «verdades» respecto de las opiniones (disponer estos saberes de modo tal que una verdad pueda traspasarlos, transformarlos, reconstruirlos, etc.). García Puchades, aludiendo a la propuesta badiouiana, indica que «la paradoja de todo esto (...) es el carácter desigualitario implícito en estas prescripciones» (2012: 254). Para García Puchades, una educación que diferencie los saberes de las verdades, implicaría necesariamente una desigualdad. No obstante, que la educación establezca tal distinción no significa en modo alguno que suponga clases de individuos «poseedores» o «no-poseedores» de verdades. Tampoco quiere decir que existan aquellos que tienen la capacidad de trascender las opiniones y elaborar una verdad, y aquellos que no gozan de esa aptitud. Como veremos, no existe en 
este aspecto, como sostiene García Puchades, paradoja alguna.

Sin embargo, el autor persiste en señalar que:

Esta desigualdad es quizás la que subyace en la crítica de Rancière al Sócrates de Platón (...) Desde nuestro punto de vista, el filósofo-militante de Badiou parte de un pseudo-saber del que no disponen aquellos que no son filósofos instaurando desde el principio una desigualdad con respecto a éstos (Ibid., 254).

En un sentido similar argumentan Love y May, en un artículo denominado «From universality to equality: Badiou's critique of Rancière» (2008). Para ellos, si bien la verdad en Badiou apelaría a una máxima universal, ese universalismo traería rápidamente consecuencias desigualitarias, al oponer entre sí a los militantes de esa verdad con el resto de los integrantes de una situación. Los autores, que omiten desarrollar en su texto la teoría de las diversas figuras subjetivas y el vínculo entre ellas propuesto por Badiou en Lógicas de los mundos, concluyen a partir de la oposición entre los militantes y el «resto», que el concepto de igualdad en Badiou, entonces, no sería un principio político sino, más bien, un resultado de la acción de los militantes. Esto lo alejaría de Rancière, quien sostiene el principio de igualdad heterogénea entre todo ser «parlante» como punto de partida:

Lo universal, a pesar de su apelación a todos, requiere de una división de la situación en dos, creando así la enemistad desde el principio entre los que juran fidelidad a la verdad y aquellos que no lo hacen, siendo los primeros teóricamente superiores porque han decidido ser sujetos de la verdad, para unirse a los elegidos» (Love y May 2008: 11). ${ }^{1}$

Esto tendría consecuencias directas sobre la noción de «igualdad» aplicada al plano educativo: según los autores, dado que existen verdades, los militantes (en oposición al resto) serían quienes las «comprenden» y quienes estarían encargados de enseñárselas a los que no lo pueden hacer. Love y May sitúan el pensamiento de Badiou dentro de un marxismo ortodoxo, admirador de Mao y de Lenin, figuras que para Badiou habrían comprendido mejor que el pueblo el suplemento acontecimiental y que, debido a ello, «atraían hacia sí mismos a los demás». En este sentido, afirman que la propuesta badiouiana asocia el militante a una figura de vanguardia opuesta a las masas.

Desde nuestra perspectiva, nada de esto podría atribuírsele al pensamiento de Alain Badiou. Existe una diferencia de perspectiva teórica entre su propia

1 Traducción propia: «The universal, despite its appeal to all, requires a division of the situation into two, thereby creating enmity from the outset between those who swear fidelity to the truth and those who do not, the former being notionally superior because they have chosen to be subjects of the truth, to join the elect». 
filosofía y la de Rancière, sobre todo, en el hecho de que éste pareciera eludir teorizar sobre una figura subjetiva específica ligada a las consecuencias ocasionadas por la inscripción de la igualdad, y por el contrario, Badiou despliega un análisis más estructurado y más amplio sobre los sujetos y sobre las consecuencias disruptivas de la declaración igualitaria. Sin embargo, esto no significa que la igualdad para Badiou no sea un principio o un axioma, al igual que lo es para Rancière. Y el hecho de que las consecuencias de la igualdad sean sostenidas por una «parte» y no por el «todo» tampoco lo distanciaría de Rancière, ¿o acaso podría pensarse que la propuesta igualitaria de Jacotot fue acogida universalmente sin encontrar resistencias en el progresismo ilustrado? De hecho, cuando Rancière describe todo el movimiento de adhesión a Jacotot y todo el contra-movimiento de resistencia a sus propuestas, está destacando diversas figuras subjetivas. La diferencia, como describiremos más adelante, es que Badiou posee una amplia teoría del sujeto y Rancière no ha creído necesario teorizar sobre ello.

Para Badiou, una verdad guarda por sí misma una relación consustancial con la igualdad. Por lo tanto, un procedimiento político fiel a una verdad lo será también con respecto a la igualdad. Los procesos de constitución de una figura subjetiva fiel no convocan a los individuos según su «comprensión» de una verdad, ni ésta es el resultado de su accionar diferencial con respecto al resto de los individuos.

Para García Puchades, Love y May, en la propuesta badiouiana del sujeto fiel existiría implícito un principio desigualitario. En primera instancia, es el propio Badiou quien refuta esa postura cuando aleja del universo del saber la figura del militante, y no lo hace en algún texto marginal, sino en una de sus obras principales, L'être et l'événement. Reiteremos: «Una fidelidad no puede depender del saber. No se trata de un trabajo sapiente sino de un trabajo militante» (Badiou 1988, 365). También podemos encontrar múltiples afirmaciones del tipo de que un filósofo no es tal por la posesión de determinados saberes, sino que lo es por medio de una actitud o de un gesto específico. Una vez más, ni el filósofo ni el militante se distinguen de los demás, en Badiou, por la cualidad de la posesión del saber.

En segundo lugar, imaginemos el hecho de que Badiou fundamente una desigualdad de origen en la posesión, ya no de un saber, sino de esa actitud crítica respecto de los saberes vinculada a la captación de verdades, y que, en ello, como afirman García Puchades, Love y May se distinguiría de Rancière. ${ }^{2}$

2 Admitamos que la diferencia con respecto al grado de estima hacia Platón de ambos autores es un punto central a tener en cuenta para todo aquel que se dedique a establecer una comparación entre ellos. Pero esta confrontación se encuentra matizada por la reinterpretación que de Platón realiza Badiou, aunque la diferencia sigue sosteniéndose marcadamente en las in- 
Tampoco sería éste el caso, ya que Rancière se ve obligado a reconocer que aquella potencia igualitaria no siempre, en toda situación, está puesta en acto. De allí la divergencia entre «inteligencia»y «voluntad», y en consecuencia, la introducción de los conceptos de «atención» y de «pereza»: la atención rancieriana sería, en cierta medida, una actitud respecto de los saberes similar a la planteada por Badiou. ${ }^{3}$ De allí, además, la figura del maestro que «fuerza» otra voluntad, aunque lo haga sin subordinar esa inteligencia. Reiteramos que esa cualidad volitiva o actitudinal es meramente circunstancial, no es una cualidad estática que determina el rol de un individuo a lo largo del tiempo (nótese la necesidad de «actualización» de la igualdad en Rancière), sino que se asume circunstancialmente y que no establece el criterio de cuenta de las situaciones educativas en las propuestas desprendidas de la teoría de Badiou. De todos modos, nada garantiza que, dado un acontecimiento que interrumpa los criterios de cuenta, la metaestructura estatal busque reordenar el estado de cosas tras la modificación de esos criterios o de esas leyes y utilice nuevos elementos como pautas diferenciadoras.

De este modo, no existirían aquellos «dotados de voluntad» frente a los que carecen de ella, o aquellos «hombres atentos» en oposición a los perezosos, o los que poseen la capacidad de comprender o acceder a las verdades y los que permanecen sumergidos en el mundo de las opiniones. Todas esas categorías no se erigen como criterios diferenciadores de clases de individuos, y por tanto, no fundan las situaciones educativas sobre una «desigualdad» como sugieren García Puchades, Love y May. Postulamos aquí que la «atención» o aquella actitud cuestionadora debe estar presente en la educación, no como un rasgo exclusivo del maestro que lo distinga de sus alumnos, sino como exigencia de la propia educación, del propio sujeto colectivo fundado en el principio igualitario.

tenciones filosóficas de cada propuesta respecto de la elección del rechazo o de la reconstrucción afirmativa del platonismo (como veremos más adelante Badiou incluye a Rancière en la lista de «antifilósofos»). Encontramos, entonces, un punto de tensión entre Badiou y Rancière sobre la figura político-pedagógica de Sócrates. Esta tensión obedece a la consideración crítica que hace Rancière de la filosofía platónica mientras que Badiou, tras una reinterpretación novedosa de La república, intenta recrearla afirmativamente. Este matiz paradójico que representa la figura de Sócrates para la educación es tratado en Kohan (2008), Filosofía, la paradoja de aprender y enseñar.

3 Si bien no son conceptos idénticos, el argumento apunta a que la igualdad de las inteligencias propuesta por Rancière requiere ser actualizada, para lo que supone la actitud de «atención». Es decir, la igualdad de las inteligencias no niega diferencias de voluntades o de actitudes como la atención y la pereza, por lo que en ello no se distanciaría considerablemente de aquella actitud subjetiva que, impulsada por un acontecimiento, buscaría atravesar los saberes instituidos (como propone Badiou). 
En una situación particular, en un momento particular, puede que algún individuo o algunos de ellos asuman más que otro/s algún rasgo propio de esas actitudes, sin embargo, eso no indica que esa situación educativa se funde, necesariamente, en un principio desigualitario. Toda situación, en la medida que es una presentación de múltiples, admite, naturalmente, diferencias. Tanto en la propuesta rancieriana como en la badiouiana, en modo alguno se parte de una desigualdad más que las que representarían algunas diferencias circunstanciales. De hecho, el proceso de construcción de una verdad, para el caso de la educación, es justamente un proceso de construcción y reconstrucción colectiva. Ese proceso dinámico de producción demanda más el ejercicio de la «participación» en el presente creador que el atributo de una "posesión» pretérita (sea de saberes o de cualidades).

Sin embargo, reconozcamos alguna sustancia en las críticas cruzadas recibidas por ambos autores de aquellos que han evaluado sus propuestas en el campo educativo. Badiou centra su atención en la distinción entre verdad y opinión, por lo que diversos autores badiouianos postulan una «educación por verdades» que nos libraría de una pedagogía que sólo sea el medio de propagación o transmisión de las opiniones. Rancière, como sabemos, fija su atención en el principio de igualdad de las inteligencias. Las acusaciones cruzadas serían las siguientes: una educación por verdades podría descuidar el principio de igualdad, y una educación igualitaria del estilo Jacotot podría quedar prisionera del mundo de las opiniones.

Reconocemos el hecho de que toda experiencia educativa (por más «marginal»o «alternativa» que se presente), en la práctica concreta pueda asumir estas características denunciadas por ambas tendencias: de hecho es frecuente en ámbitos militantes violar el principio de igualdad intelectual en nombre de una liberación ideológica, y en ámbitos horizontalistas propagar la sujeción a las opiniones dominantes en nombre de un espontaneísmo esperanzado. Pero hemos argumentado en contra de estas posturas en tanto afirmamos que no se desprenden, necesariamente, de las filosofías de ambos autores.

Como ya señalamos, no se concluye de una «educación por verdades» ningún principio desigualitario, ya que el proceso de construcción de verdades está ligado a un requerimiento dinámico del presente acontecimiental a través de un procedimiento subjetivo de fidelidad en el que cualquiera podría participar sin requisitos de posesiones previas (mucho menos del género del saber). Recordemos que una característica específica de las verdades en Badiou era su universalismo radical, el estar constituidas por el «para-todos» igualitario. Del mismo modo, no se desprende de la educación igualitaria rancieriana su empantanamiento en el círculo de la reproducción de las opiniones, ya que en ella se considera una actitud (por caso, la «atención») y la participación en una potencia intelectual y creativa que supondría la superación de la mera 
incorporación y repetición de opiniones ya constituidas.

Badiou mismo admite, en uno de sus recientes cursos, la cercanía de Jacotot con la de su propio Platón:

l'éducation est une affaire d'orientation: 'il importe seulement de trouver le moyen le plus simple et le plus efficace pour que s'opère (le) retournement du Sujet'. Il s'agit de réorienter une faculté que le Sujet a déjà (la doctrine de la réminiscence telle qu'elle est exposée dans l'épisode de l'esclave du Ménon est ici fondamentale). On voit à cette occasion la proximité de Platon avec le Jacotot de Le Maître ignorant qui n'est donc pas si anti-platonicien que le dit Jacques Rancière (2008-09, 20 de mayo 2009). ${ }^{4}$

\section{ENCUENTROS Y DESENCUENTROS FILOSÓFICOS Y POLÍTICOS EN LA EDUCACIÓN}

Rancière retoma y continúa algunos aspectos de la obra foucaultiana, específicamente la relación entre saber y poder. Tal es así, que en su libro sobre Althusser (1974), se ocupa de la relación entre los saberes de la ciencia althusseriana y la autoridad política del Partido Comunista francés.

En realidad, estas temáticas se imponían en el contexto de la década de 1960, época heredada por ambos autores. Son esos años los que testimonian el cambio entre la hegemonía de una postura cientificista (el saber del intelectual) a una que prioriza la praxis de la acción (de los actores directos). En un primer momento, el dispositivo principal de las ciencias humanas era cierto neocientificismo de corte estructuralista gobernado por Althusser y Lacan. Era una época en la que la cuestión del saber resultaba elemental.

No obstante, hacia mediados de los años sesenta, esta tendencia comienza a revertirse. La Revolución Cultural china ubica en el centro de las discusiones la cuestión de la autoridad del saber. En este sentido, podremos mencionar las revueltas estudiantiles antijerárquicas contra autoridades académicas y las revueltas fabriles contra los jefes que ostentaban la posesión de los conocimientos técnicos a través de los cuales legitimaban la ocupación de los cargos superiores. Estas revueltas que cuestionaban la autoridad del saber destacaban, en cambio, la experiencia directa de obreros y estudiantes.

A los sucesos chinos se sumaron los acontecimientos franceses de fines

4 «La educación es una cuestión de orientación: es importante solamente encontrar el recurso más simple y eficaz para que se produzca una transformación [o una conversión] radical del Sujeto. También es una opción para reorientar una facultad que el Sujeto ya posee (la doctrina de la reminiscencia como está expuesta en el episodio del esclavo de Menón es fundamental aquí). Vemos en esta ocasión la proximidad de Platón con el Jacotot de El Maestro ignorante, que no es tan antiplatónico como dice Jacques Rancière» (2008-09: 20 de mayo 2009, traducción propia). 
de la década de 1960. Las revueltas de los jóvenes obreros en fábricas francesas apuntaban contra las jerarquizaciones sindicales y contra toda forma de autoridad interna. A esto se suma, además, los episodios conocidos de Mayo de 1968, que se dirigen de manera directa en oposición a la organización vertical de la transmisión de los saberes.

Tenemos así dos grandes paradigmas. El reinado de los saberes científicos y su estructura jerárquica dominada por los docentes-científicos, y la denuncia contra la opresión dominante ubicada en el vínculo entre saber y autoridad. En este contexto, en el que era difícil evitar la cuestión del saberpoder, es en el que se ubican tanto Jacques Rancière como Alain Badiou. El problema fundamental, entonces, para ellos, era pensar la educación una vez destituida la figura de la autoridad del saber.

En la primavera de 2005, Badiou pronuncia un discurso sobre Jacques Rancière, en un coloquio dedicado a este último en Cerisy. En él analiza la destitución de la autoridad del saber y le adjudica a Rancière inclinarse por una hipótesis democrática. La irrupción del movimiento, de la multitud, sirve de fundamento para un nuevo tipo de transmisión y de maestría. Es por eso que se ve obligado a adoptar en sus investigaciones un carácter historicista. De allí la ocupación por el tema del «reparto» o de la «partición» (partage): de poderes, de saberes, de lo visible, etc. La destitución de la autoridad del saber es consecuencia del cambio producido por la inscripción de una parte que antes era invisibilizada.

Badiou, marca una leve distancia de esta posición, a la que autodenomina (a través de una provocadora ironía) como «aristocrática». La nueva educación, para él, se vincula directamente con los efectos acontecimientales de un cuerpo heterogéneo. Este cuerpo afecta de manera diversa al demos. Señalemos una vez más, que esta afectación diferencial no refiere a criterios del tipo de posesión de saberes previos o de habilidades adquiridas. Esta diferencia no afecta el principio de igualdad compartido por ambos, sino que es, más bien, una distinción de «perspectiva»: refiere a qué aspectos por sobre otros cada autor le ha otorgado mayor interés. Badiou acusa a Rancière, nuevamente, de no profundizar en los medios de organización de las consecuencias de lo que podría sugerir la ruptura con las formas de transmisión preestablecidas. Ante la necesidad de nombrar ese cuerpo heterogéneo que se encargaría de la militancia respecto de la duración de la igualdad, es que Badiou opone a la figura del maestro ignorante, la de una aristocracia proletaria. Tal «aristocracia» es completamente contingente y abierta a la participación de cualquiera.

Una vez más, encontramos que un aspecto central de esta divergencia, es el vínculo que tiene cada uno con Platón. Como es sabido, éste ha tratado insistentemente, sobre todo en República, la conexión entre la disposición del saber y la distribución de los lugares de poder. Mientras que Rancière fija su 
atención en el aspecto de la repartición social platónica, Badiou lo hace en referencia a la educación de los guardianes. En el primer caso, Rancière repara en la univocidad de tareas del artesano (la prescripción platónica lo fuerza a ocuparse de una única tarea) que lo subordina en la distribución de los lugares sociales. En el segundo, Badiou se concentra en los propios guardianes, los jefes políticos, quienes tienen prescriptos para sí una multiplicidad de ocupaciones (aunque alejadas del universo del trabajo manual, constituyen una variedad). Badiou trasciende el plano social en un esfuerzo teórico provocador para hacer de los guardianes una figura universal (humanidad genérica o sin clases) y fuerza a Platón a inscribirse en un paradigma comunista.

Ahora bien, Badiou reconoce que ambos sostienen la idea, extraída del maoísmo, de lucha en dos frentes. Esta lucha en política significaba originariamente una activación por igual contra la posición burguesa, capitalista e imperialista, y contra el partido comunista y el sindicalismo institucional. Pero el concepto de lucha en dos frentes aplicado a la cuestión específica del saber-poder, hacía que tanto Badiou como Rancière se opongan por igual a: i- la ciencia como fundamento de la política y que, por tanto, debía ser transmitida por los expertos del Partido a los obreros ignorantes; ii- la política como espontaneidad o como vitalismo de las masas, ajena a las ideas.

En referencia al primer aspecto (i), ambos autores rompen, al mismo tiempo, con su maestro Louis Althusser. Éste vendría a representar la figura del maestro-sabio, quien aboga por la idea de que la «conciencia» al obrero le llega «desde afuera», ${ }^{5}$ desde la ciencia positiva que no es otra que el marxismo. En lo que hace al segundo frente, ambos desligan a la política de cualquier carácter vitalista o espontáneo, resaltando su aspecto declarativo y prescriptivo: ambos afirman que la política es un «pensamiento», con la misma carga de ruptura con respecto a la reproducción de las opiniones. Esto se traduce en el ámbito de la educación, a que si bien se enfrentan al maestro-sabio y a la transmisión unilateral de saberes instituidos, no renuncian a todo tipo de educación y a todo tipo de actividad productora de ideas y conceptos.

Las críticas cruzadas de los comentadores de ambos autores en el ámbito de la educación quedan expuestas a su inconsistencia con la hipótesis de la lucha en dos frentes. Ni Badiou descuida la igualdad (primer frente contra el maestro-sabio), ni Rancière descuida el aspecto crítico de trascender las opiniones (segundo frente contra el espontaneísmo educativo).

La educación rancieriana, a través de conceptos tales como «emancipación», «igualdad de las inteligencias», «maestro ignorante», «comunidad de los iguales», rompe con la conexión platónica entre el maestro-sabio y el dirigente de la ciudad. Esto desplaza y redefine el estatuto de los «saberes»

5 Lo que los maoístas llamaban «leninismo osificado». 
puestos en juego en la relación igualitaria, y que asociamos con el concepto de «verdades» propuesto por Badiou:

El saber en tanto se encuentra bajo la condición de la máxima igualitaria, en una nueva relación con la ignorancia y que abre a su vez un nuevo lugar para la igualdad, es un saber evidentemente desplazado respecto del saber instituido. En mi propia jerga, esto querría decir que obtenemos un saber a la altura por lo menos de una verdad (Badiou 2012b, 256).

Sin embargo, entre estas coincidencias, Badiou reitera una diferencia elemental. Señala que Rancière se detiene justo allí en donde la igualdad se ha manifestado, y eso lo excusa de poseer una teoría de las consecuencias de lo que ella ha provocado. Badiou posee una teoría del acontecimiento, de la fidelidad, de la constitución de un cuerpo-de-verdad y de la composición de las figuras subjetivas. Todas esas cuestiones, ligadas a la «organización» de las disrupciones son las que Badiou reclama inconclusas en Rancière. Si bien éste recusa la organización impuesta por el Partido o por el Maestro-Sabio, pone en suspenso el principio organizacional mismo, con posibles derroteros políticos y filosóficos que lo alejan en algún aspecto de Badiou.

Para recapitular, el acuerdo elemental entre Badiou y Rancière se sostiene en el concepto de igualdad en tanto declaración, y nunca asumido de forma programática. Esto, en el ámbito educativo, anula la pregunta de quién debe educar a quién. Por lo que postulamos que el sujeto de la educación es una figura colectiva. El acuerdo fundamental nos provee una fórmula que afirma que «el proceso anónimo de la educación es la construcción de un colectivo compuesto por las consecuencias de una declaración igualitaria situada» (Ibid., 257). El problema denominado «¿quién educa a quién?» se halla desplazado por el carácter anónimo del «nosotros nos educamos», propio del sujeto colectivo.

Efectivamente, de este acuerdo sustancial proviene el desacuerdo primario: la delimitación del «nosotros». Pareciera no hallarse en Rancière ningún tipo de prescripción que circunscriba al sujeto, a la figura militante. Por el contrario, Badiou (2006) elabora una amplia teoría con respecto al «nosotros» (teoría de los campos subjetivos y de los cuerpos de verdad), aunque lo hace sin referirse al ámbito específico de la educación, y concentrado más bien en el campo político. 


\section{REFERENCIAS BIBLIOGRÁFICAS}

BAdiou, A. 2012a. «Acontecimiento y subjetivación política». En López, M. (ed.) La hipótesis comunista. Recuperado desde http://lahipotesiscomunista.blogspot. com.ar/2013/07/acontecimiento-y-subjetivacion-politica.html: diciembre 2013.

BADiou, A. 2012b. L'aventure de la philosophie française. Paris: La Fabrique. [Citas de la traducción al español de Agoff, I., La aventura de la filosofía francesa a partir de 1969, Buenos Aires, Eterna Cadencia, 2013].

Badiou, A. 2008-09. Pour aujourd'hui: Platon! (2), transcripción de Daniel Fischer, disponible en: < http://www.entretemps.asso.fr/Badiou/08-09.htm $>$ [consultado 09-2013].

Badiou, A. 2006. Logiques des mondes. L'être et l'événement, 2. París: Seuil.

Badiou, A. 1998. Abrégé de métapolitique. París: Seuil. [Citas de la traducción al español de Spinelli, J., Compendio de metapolítica, Buenos Aires, Prometeo, 2009].

BAdiou, A. 1988. L'être et l'événement. París: Seuil. [Citas de la traducción al español de Cerdeiras, R., Cerletti, A. y Prados, N., El ser y el acontecimiento, Buenos Aires, Manantial, 1999].

García Puchades, W. 2012. El lugar del cine en el pensamiento filosófico de Alain Badiou. Tesis de doctorado, Facultad de Bellas Artes de San Carlos, Universidad Politécnica de Valencia, España. Recuperada en http://riunet.upv.es/bitstream/ handle/10251/14862/tesisUPV3751.pdf?sequence=1\&isAllowed=y

KoHAn, W. 2008. Filosofía, la paradoja de aprender y enseñar. Buenos Aires: Libros del Zorzal.

Love. J. Y MAY, T. 2008. «From universality to equality: Badiou's crituques of Rancière», en Symposium: Canadian Journal of Continental Philosophy, vol. 12, núm. 2, pp. 51-69.

RANCIÈRE, J. 2004. «El uso de las distinciones». Conferencia impartida en la jornada «Partición de lo sensible» en el Colegio Internacional de Filosofía, organizada por Jean-Clet Martin. Publicada en Failles, núm. 2, 2006. Recuperado de http:// www.ddooss.org/articulos/otros/J_Ranciere.htm

Rancière, J. 1995. Le Mésentente. Politique et philosophie. Paris: Galilée.

RANCIÈRE, J. 1987. Le maître ignorant: Cinq leçons sur l'émancipation intellectuelle. París: Fayard.

RAnCiÈRe, J. 1974. La Leçón d'Althusser. París: Gallimard. 
Leonardo Colella es investigador en el Consejo Nacional de Investigaciones Científicas y Técnicas (CONICET). Facultad de Filosofía y Letras, Universidad de Buenos Aires (FFyL - UBA).

Linea de investigación:

Igualdad y sujeto en la educación, a partir de los aportes de las filosofías de Alain Badiou y Jacques Rancière.

Publicaciones:

"Foucault y Rancière como educadores. La explicación como técnica de sí mismo" en Revista Sul-Americana de Filosofía e Educação (ISSN 1679-8775), núm. 18 (Brasilia: mayooctubre de 2012), pp. 173-184.

"Las coronas de la educación. Subjetividad y reproducción en las instituciones educativas del siglo XXI" en Revista Paideía (ISSN 0214-7300), XXXII, 96, Madrid: septiembre 2012, pp. 281-288.

Correo electrónico:

leonardojcolella@yahoo.com.ar 